\title{
Der Europäische Vollstreckungstitel in C2C-Streitigkeiten
}

\section{Zugleich Anmerkung zu EuGH, Urt. v. 5.12.2013, Rs. C-508/12 Vapenik ./. Thurner}

\author{
Matthias Klöpfer/Paša Ramić, Konstanz
}

\section{A. Einleitung}

Die vorliegender Abhandlung zugrunde liegende Entscheidung des EuGH in der Rechtssache Vapenik ${ }^{1}$ erging zur Auslegung von Art. 6 Abs. 1 lit. d der Europäischen Vollstreckungstitel-Verordnung (EuVTVO $)^{2}$ und der im deutschsprachigen Schriftum umstrittenen Frage, ob bei Streitigkeiten zwischen Verbrauchern ( $\mathrm{C} 2 \mathrm{C}=$ consumer to consumer $)$ nur solche Entscheidungen als Europäischer Vollstreckungstitel bestätigt werden können, die im Wohnsitzmitgliedstaat des verklagten Verbrauchers ergangen sind. ${ }^{3}$ Mit der EuVTVO ist seit Oktober 2005 die Bestätigung bestimmter unbestrittener ${ }^{4}$ gerichtlicher Entscheidungen als Europäischer Vollstreckungstitel möglich, womit nach Art. 5 EuVTVO die unmittelbare Zwangsvollstreckung in allen Mitgliedstaaten ermöglicht wird, ohne dass es eines Exequaturverfahrens im Vollstreckungsstaat bedarf. ${ }^{5}$ Vorliegende Entscheidung stellt hierbei erst die zweite Präzisierung der EuVTVO durch den EuGH dar und ist schon alleine deshalb von besonderem Interesse. ${ }^{6}$ Bedeutung kommt ihr allerdings für das gesamte Europäische Zivilverfahrensrecht zu und dies aus mehreren Gründen: Der EuGH wird in Auslegung von Art. 6 Abs. 1 lit. d EuVTVO rechtsaktübergreifend ${ }^{7}$ tätig, bezieht demnach andere europäische Rechtsakte EuGVVO, ${ }^{8}$ Rom I-VO ${ }^{9}$ sowie die Klauselrichtlinie ${ }^{10}$ - in die Auslegung bzw. zur Begründung seines Auslegungsergebnisses ein. In der Sache ist dies, mit Blick auf den Stand einer Europäischen Methodenlehre, ${ }^{11}$ von Bedeutung. Daneben steht im Zentrum der Entscheidung die Frage nach der personellen Reichweite von verbraucherschützenden Vorschriften im Europäischen Zivilverfahrensrecht. Damit wirkt die Entscheidung auf die Auslegung ebensolcher Vorschriften im sonstigen Europäischen Zivilverfahrensrecht zurück, was auch der konkret durch den EuGH bemühten Methodik geschuldet ist. Der Entscheidungsinhalt ist daher insbesondere mit Blick auf die Art. 15 ff. EuGVVO, das insoweit parallel liegende Luganer Übereinkommen, ${ }^{12}$ aber auch mittelbar bei Anwendung der Europäische Mahnverordnung ${ }^{13}$ und der Verordnung über ein Verfahren für geringfügige Forderungen $^{14} \mathrm{zu}$ berücksichtigen.

\section{Die Gefahren der EuVTVO für Verbraucher}

Die Bestätigung einer unbestrittenen gerichtlichen Entscheidung als Europäischer Vollstreckungstitel birgt für den Titelschuldner,

EuGH, Urt. v. 5.12.2013, Rs. C-508/12 - Vapenik./. Thurner, ZIP 2014, 100 (Leitsatz), im Volltext bei BeckRS 2013, 82273.

2 Verordnung (EG) Nr. 805/2004 des Europäischen Parlaments und des Rates vom 21. April 2004 zur Einführung eines Europäischen Vollstreckungstitels für unbestrittene Forderungen, ABl. L 143 v. 30.4.2004, S. 15.

3 Dagegen: Bach, Grenzüberschreitende Vollstreckung in Europa, Tübingen 2008, S. 220 f.; Geimer, in: Geimer/Schütze, Europäisches Zivilverfahrensrecht, 3. Aufl. 2010, Art. 6 VO (EG) Nr. 805/2004 Rn. 9; ders., in: Zöller, 29. Aufl. 2012, Art. 6 EG-VO Europ. Vollstreckungstitel Rn. 3; Hess, Europäisches Zivilprozessrecht, 2010, $\$ 10$ Rn. 18.; i.E. van Husen, Gerichtsstand in Verbraucherangelegenheiten im Österreichischen und Europäischen Zivilprozessrecht, Wien 2009, abrufbar unter: http://othes .univie.ac.at/8527/(zuletzt abgerufen am 10.2.2014), S. 158 f., mit allerdings sinnwidrigen Ausführungen und Nachweisen; Kropholler/von Hein, Europäisches Zivilprozessrecht, 9. Aufl. 2011, Art. 6 EuVTVO Rn. 13; Mankowski, in: FS Karameus, S. 785, 796 f.; ders., VuR 2010, 16, 21; wohl auch Schlosser, EU-Zivilprozessrecht, 3. Aufl. 2009, Art. 6 VTVO Rn. 6. Dafür: Coester-Waltjen, in: FS Yessiou-Faltsi (Athen 2007), S. 39, 43; Pabst, in: Rauscher, EuZPR/EuIPR, Bearbeitung 2010, Art. 6 EG-VollstrTitelVO Rn. 40; Rauscher, IPRax 2011, 484, 486; Halfmeier, in: Prütting/Gehrlein, ZPO, 4. Aufl. 2012, Art. 6 VO 805/2004 Rn. 5; Ringwald, Europäischer Vollstreckungstitel nach der EuVTVO und Rechtsbehelfe des Schuldners, Baden-Baden 2011, S. 51; M. Stürner, in: Kindl/MellerHannich/Wolf, Gesamtes Recht der Zwangsvollstreckungsrecht, 2. Aufl. 2012, Art. 6 EuVTVO Rn. 8; wohl auch Garber, Einstweiliger Rechtsschutz nach der EuGVVO, Diss., Wien u.a. 2011, S. 296 und Heringer, Der Europäische Vollstreckungstitel für unbestrittene Forderungen, Baden-Baden 2007, S. 117. Unklar: Stein, IPRax 2004, 181, 189.

4 Vgl. hierzu Art. 3 Abs. 1 EuVTVO.

5 Allgemein zur EuVTVO, vgl. Heringer (s.o., Fn. 3); Ringwald (s.o., Fn. 3); Rauscher (s.o., Fn. 3); Coester-Waltjen, Jura 2005, 394 ff.; Giebel, IPRax 2011, 529 ff. Speziell zum Verbraucherschutzniveau im Rahmen der EuVTVO, vgl. Mankowski, VuR 2010, 16 ff.

6 Vgl. die andere Entscheidung des EuGH zur EuVTVO, Urt. v. 15.3.2012, Rs. C-292/10 - G./. Cornelius de Visser, EuZW 2012, 381, zur Frage, ob bei unbekanntem Wohnsitz die Bestätigung eines Urteils als Europäischer Vollstreckungstitel erfolgen kann.

7 Zur sog. rechtsaktübergreifenden Auslegung im Europäischen Zivilverfahrensrecht, vgl. Lüttringhaus, RabelsZ 77 (2013), 31 ff.; Rühl, GPR 2013, $122 \mathrm{ff}$. 
insbesondere aber für Verbraucher, eine erhebliche Gefahr: Sie führt zu einer Erhöhung der Prozessführungslast im Ausland. ${ }^{15}$ Typischerweise vermeiden Verbraucher jedoch Prozesse im Ausland und versuchen nicht selten, Verfahren durch bloßes Abwarten auszusitzen. ${ }^{16}$ Säumnisentscheidungen sind in Fällen der Beteiligung eines Verbrauchers gerade nicht unüblich; genau diese hat aber die EuVTVO als Bezugspunkt. ${ }^{17}$ Wegen des Wegfalls eines zwischengeschalteten Exequaturverfahrens bei Bestätigung einer Entscheidung nach der EuVTVO (vgl. Art. 5 EuVTVO) droht für den Titelschuldner die unmittelbare Zwangsvollstreckung und dies theoretisch parallel in mehreren Mitgliedstaaten. Auch wenn Verbraucher in aller Regel Vermögenswerte innerhalb ihres Wohnsitzmitgliedstaates bündeln werden und damit die Vollstreckung in mehreren Mitgliedstaaten für sie keine erhebliche Gefahr darstellt, ${ }^{18}$ sind es die nach der EuVTVO drastisch beschränkten Rechtsbehelfsmöglichkeiten, die gerade sie benachteiligen. Neben den klassischerweise im Ursprungsmitgliedstaat geltend zu machenden Einwendungen gegen den Titel selbst, bündelt die EuVTVO nämlich die durch Art. 10 EuVTVO auch in der Sache beschränkten Einwendungen ${ }^{19}$ gegen die Bestätigung im Ursprungsmitgliedstaat ${ }^{20}$ und zwingt Verbraucher damit, in jedem Fall im Ausland zu prozessieren. ${ }^{21}$ Die hiermit zusammenhängenden Belastungen werden Privatpersonen häufig nicht auf sich nehmen wollen; es steht zu befürchten, dass so die ohnehin begrenzten Rügemöglichkeiten unter Umständen gar nicht genutzt werden. Im Vollstreckungsmitgliedstaat selbst besteht lediglich die Möglichkeit, den Widerspruch zu einem früheren Titel vorzubringen. ${ }^{22}$

Insgesamt zeigt sich hierin ein bemerkenswerter Unterschied zur grenzüberschreitenden Vollstreckung sonstiger gerichtlicher Entscheidungen in Zivilsachen, die nicht nach der EuVTVO bestätigt werden, sondern gleichsam „klassisch“ im Ausland vollstreckt werden sollen. Zwar wird für diese mit dem vollständigen Inkraftreten der reformierten EuGVVO ${ }^{23}$ zum 10.1.2015 ebenfalls kein zwischengeschaltetes Vollstreckbarerklärungsverfahren mehr notwendig sein, ${ }^{24}$ allerdings konnte im Reformprozess gegen erheblichen Widerstand aus den Mitgliedstaaten gerade nicht durchgesetzt werden, die Rechtsbehelfe des Titelschuldners in den Ursprungsmitgliedstaat zu verlagern oder in der Sache zu kürzen; eine Fortführung des in der EuVTVO angestoßenen Projekts war gerade nicht vermittelbar. ${ }^{25}$ So wird es unter der reformierten EuGVVO auch mit Wegfall des Exequaturverfahrens für einen Schuldner weiterhin möglich sein, die derzeit noch in den Art. 34, 35 EuGVVO festgeschriebenen Einwendungen im Vollstreckungsmitgliedstaat geltend zu machen, wobei diese sogar noch erweitert wurden um die Rüge der Verletzung des Zuständigkeitsregimes in Arbeitssachen. ${ }^{26}$ Alleine der Zeitpunkt wird sich ändern: Mangels Vollstreckbarerklärungsverfahren sind die Einwendungen mittels eines Rechtsbehelfs im Zwangsvollstreckungsverfahren vorzubringen. ${ }^{27}$

\section{Verbraucherschutz in der EuVTVO}

Vor diesem Hintergrund gewinnt der in der EuVTVO verbriefte Verbraucherschutz eine erhebliche Bedeutung. ${ }^{28}$ Neben Mindestvorschriften zur Zustellung und anderen Voraussetzungen der Bestätigung, die faktisch schützen wirken, ist hier vor allem
Art. 6 Abs. 1 lit. d EuVTVO zu nennen. Im Gegensatz zu Verbraucherschutz über eine zuständigkeitsrechtliche Primärebene, wie er etwa in Art. 15 ff. EuGVVO ${ }^{29}$ zu finden ist, betrifft Art. 6 Abs. 1 lit. d EuVTVO und demnach auch die Entscheidung des EuGH in Vapenik gleichsam eine sekundäre Ebene des Verbraucherschutzes: Gegenüber einem Verbraucher als Titelschuldner findet die Bestätigung einer gerichtlichen Entscheidung als Europäischer Vollstreckungstitel nach Art. 6 Abs. 1 lit. d EuVTVO nur statt, wenn sie in dessen Wohnsitzmitgliedstaat erstritten wurde. ${ }^{30}$ Die Einhaltung dieser Voraussetzung hat das bestätigende Gericht unabhängig vom Ergebnis der Zuständigkeitsprüfung durch das titelschaffende Gericht zu gewährleisten. ${ }^{31} \mathrm{Im}$ Unterschied zu Entscheidungen in Arbeits- und Versicherungs-

8 Verordnung (EG) Nr. 44/2001 des Rates vom 22. Dezember 2000 über die gerichtliche Zuständigkeit und die Anerkennung und Vollstreckung von Entscheidungen in Zivil- und Handelssachen, ABl. L 12 v. 16.1.2001, S. 1.

9 Verordnung (EG) Nr. 593/2008 des Europäischen Parlaments und des Rates vom 17. Juni 2008 über das auf vertragliche Schuldverhältnisse anzuwendende Recht (Rom I), ABl. L 177 v. 4.7.2008, S. 6.

10 Richtlinie 93/13/EWG des Rates vom 5. April 1993 über missbräuchliche Klauseln in Verbraucherverträgen, ABl. L 95 v. 21.4.1993, S. 29).

11 Vgl. Fleischer, RabelsZ 75 (2011), 700, 708 ff.; Höpfner/Rüthers, AcP 209 (2009), 1, 2: sprechen von einem zeitweiligen „methodische(n) Vakuum“; Rösler, Rechtstheorie 43 (2012), 495, 497 m. umfangreichen Nachw.

12 Luganer Übereinkommen über die gerichtliche Zuständigkeit und die Anerkennung und Vollstreckung von Entscheidungen in Zivil- und Handelssachen vom 30. Oktober 2007, ABl. L 147, S. 5.

13 Die Art. 15 ff. EuGVVO gelten auch im Rahmen der Europäischen Mahnverordnung, vgl. Art. 6 Abs. 1 der Verordnung (EG) Nr. 1896/2006 des Europäischen Parlaments und des Rates vom 12. Dezember 2006 zur Einführung eines Europäischen Mahnverfahrens, ABl. L 399 v. 30.12.2006, S. 1.

14 Zwar findet sich in der Verordnung (EG) Nr. 861/2007 des Europäischen Parlaments und des Rates vom 11. Juli 2007 zur Einführung eines europäischen Verfahrens für geringfügige Forderungen, ABl. L v. 31.7.2007, S. 1 kein Verweis auf das Zuständigkeitsrecht der EuGVVO, über dessen Geltung besteht aber Einigkeit, vgl. Schlosser (s.o., Fn. 3), Art. 4 BagatellVO Rn. 3.

15 Kropholler/von Hein (s.o., Fn. 3), Art. 1 EuVTVO Rn. 5 m.w. N. Vgl. auch Stadler, IPRax 2004, 2, 9; dies., RIW 2004, $801 \mathrm{ff}$.

16 Mankowski, VuR 2010, 16, 17.

17 Mankowski, VuR 2010, 16, 16. Vgl. Art. 3 Abs. 1 l. d EuVTVO, der in Verbindung mit Erwägungsgrund Nr. 6 eben auch die klassischen Fälle eines Versäumnisurteils erfasst.

18 Kropholler/von Hein (s.o., Fn. 3), Art. 6 EuVTVO Rn. 14 auch mit Nachweisen zur abweichenden Ansicht.

19 Rauscher, Der Europäische Vollstreckungstitel für unbestrittene Forderungen, 2004, Rn. 39: „rechtsstaatlich schwer zu akzeptieren.“

20 Vgl. Art. 10 Abs. 1 und 4 EuVTVO.

21 Vgl. Stadler, RIW 2004, 801, 803.

22 Vgl. Art. 21 EuVTVO. Hierzu Rauscher (s.o., Fn. 19), Rn. 178.

23 Verordnung (EU) Nr. 1215/2012 des Europäischen Parlaments und des Rates vom 12. Dezember 2012 über die gerichtliche Zuständigkeit und die Anerkennung und Vollstreckung von Entscheidungen in Zivil- und Handelssachen, ABl. L 351 v. 20.12.2012, S. 1 (im Folgenden: EuGVVO n. F.).

24 Vgl. Art. 39 EuGVVO n. F.

25 Vgl. von Hein, RIW 2013, 97, 109; Pohl, IPRax 2013, 109, 112 ff.

26 Vgl. Pohl, IPRax 2013, 109, 113.

27 Vgl. Art. 46 EuGVVO n. F.

28 Hierzu allgemein Mankowski, VuR 2010, $16 \mathrm{ff}$.

29 Klagen eines Unternehmers gegen einen Verbraucher sind unter den Voraussetzungen des Art. 15 EuGVVO nur im Wohnsitzmitgliedstaat des Verbrauchers zulässig, vgl. Art. 16 Abs. 2 EuGVVO, im selben Zusammenhang aber Aktivklagen des Verbrauchers gegen einen Unternehmer auch vor den Gerichten seines Wohnsitzes, Art. 16 Abs. 1 2. Var. EuGVVO.

30 Vgl. hierzu speziell Rauscher (s.o., Fn. 19), Rn. 93.

31 Str., vgl. Geimer, in: Zöller (s.o., Fn. 3), Art. 6 EG-VO Europ. Vollstreckungstitel Rn. 6 m.w.N. Pabst, in: Rauscher, EuZPR/EuIPR (s.o., Fn. 3), 
sachen, deren Bestätigungsfähigkeit über lit. b der Vorschrift direkt an eine Einhaltung der jeweiligen EuGVVO-Zuständigkeiten geknüpft wurde, wird für Entscheidungen, die gegen einen Verbraucher ergangen sind, in lit. d ein selbstständiger Prüfungspunkt aufgestellt.

Einigkeit besteht dahingehend, dass hiermit der Schutz von Verbrauchern erhöht werden sollte. ${ }^{32} \mathrm{Ob}$ dies von der Person des Vertragspartners abhängen soll, ist hingegen nicht offensichtlich und vor allem deshalb fraglich, da im Gegensatz zu Versicherungs- und Arbeitssachen gerade nicht auf die entsprechenden EuGVVO-Vorschriften Bezug genommen wurde, welche ausweislich Erwägungsgrund Nr. 13 EuGVVO ihre Rechtfertigung in einem Machtgefälle zwischen den betroffenen Parteien finden. ${ }^{33}$ Daher wurde dem EuGH in der Rechtssache Vapenik durch das Landesgericht Salzburg die Frage vorgelegt, ob die Schutzvorschrift des Art. 6 Abs. 1 lit. d EUVTVO die Beteiligung eines Unternehmers voraussetze (B2C $=$ business to consumer) oder auch in $\mathrm{C} 2 \mathrm{C}$-Streitigkeiten einschlägig sei.

\section{B. Sachverhalt}

Die Vorlage des Landesgerichts Salzburg erfolgte im Rahmen eines Rechtsbehelfsverfahrens, das der in Österreich wohnhafte Kläger und Antragssteller Herr Vapenik gegen die Ablehnung seines Antrags auf Ausstellung eines Europäischen Vollstreckungstitels nach der EuVTVO für ein Versäumnisurteil eingeleitet hatte. Letzteres war gegen den in Belgien wohnhaften Beklagten Herrn Thurner durch das Bezirksgericht Salzburg wegen einer Forderung aus einem Darlehnsvertrag erlassen worden. Der Darlehensvertrag war nach dem Vortrag des Klägers von den Parteien mit einer ausschließlich privaten Zwecksetzung geschlossen worden. Das Bezirksgericht Salzburg hatte die Bestätigung der Entscheidung als Europäischen Vollstreckungstitel jedoch mit der Begründung abgelehnt, sie entspreche nicht den Vorgaben des Art. 6 Abs. 1 lit. d EuVTVO. Nach dieser Vorschrift könnten nur solche Entscheidungen gegen einen Verbraucher als Europäischer Vollstreckungstitel bestätigt werden, die im Wohnsitzstaat des beklagten Verbrauchers erwirkt worden seien. Da das Urteil gegen Herrn Thurner nicht an seinem Wohnsitz in Belgien, sondern in Salzburg erstritten worden war, sei die Bestätigung zu versagen. ${ }^{34}$

\section{Entscheidung des EuGH}

Der EuGH sah dies anders. Dass die Voraussetzungen des Art. 6 Abs. 1 lit. d EuVTVO in C2C-Streitigkeiten nicht einzuhalten sind, scheint in seinen Augen derart offensichtlich gewesen zu sein, dass man auf die Zuziehung des Generalanwalts Cruz Villalón nach dessen Anhörung verzichtete. ${ }^{35}$ Der Gerichtshof hält den durch Art. 6 Abs. 1 lit. d EuVTVO vermittelten Schutz in C2C-Streitigkeiten nicht für erforderlich, womit gerade auch die Bestätigung von Entscheidungen als Europäischen Vollstreckungstitel möglich ist, die gegen Verbraucher außerhalb ihres Wohnsitzmitgliedstaats erstritten wurden. ${ }^{36}$ Stehe dem verklagten Verbraucher ein ebenfalls als Verbraucher handelnder Kläger gegenüber, bedürfe es des durch diese Vorschrift vermittelten besonderen Schutzes nicht. ${ }^{37}$ Dies folgert der EuGH insoweit aus einem Vergleich mit anderen verbraucherschützenden Vorschriften europäischen Ursprungs. Er verweist auf die Klauselrichtlinie, deren besondere Regelungen auf dem Gedanken einer Informationsasymmetrie und einer schwächeren Verhandlungsposition des Verbrauchers gegenüber einem Unternehmer beruhe. ${ }^{38}$ Auch aus dem 13. Erwägungsgrund der EuGVVO folge, dass die entsprechenden verbraucherschützenden Regelungen der Verordnung dem Schutz des wirtschaftlich Schwächeren und rechtlich weniger Erfahrenen zu dienen bestimmt seien. ${ }^{39}$ Gleiches gelte für Art. 6 Rom I-VO. ${ }^{40}$ Weiter verweist der Gerichtshof auch auf die Entscheidung in der Rechtssache Shearson Lehman Hutton, ${ }^{41}$ wo er entschieden habe, dass ein zwischen zwei Unternehmern geschlossener Vertrag nicht in den Anwendungsbereich der Regeln über den Verbrauchergerichtsstand des EuGVÜ falle, da sie deren Schutz nicht bedürften. ${ }^{42}$ Gleiches gelte für einen in einem C2C-Verhältnis abgeschlossenen Vertrag. ${ }^{43}$ Zur Abrundung führt der Gerichtshof noch ins Feld, eine Beschränkung der EuVTVO in C2C-Konstellationen auf Entscheidungen, die im Wohnsitzmitgliedstaat des verklagten Verbrauchers ergangen seien, führe zu Ungereimtheiten mit dem Vollstreckbarerklärungssystem nach den Art. $38 \mathrm{ff}$. EuGVVO: Würde man die EuVTVO in C2C-Konstallationen auf Entscheidungen beschränken, die im Wohnsitzmitgliedstaat des verklagten Verbrauchers ergangen seien, stünde dem klagenden Verbraucher dennoch die Möglichkeit offen, eine Vollstreckbarerklärung gem. Art. 38 EuGVVO im Wohnsitzmitgliedstaat des Schuldnerverbrauchers zu erwirken. ${ }^{4}$

Art. 6 EG-VollstrTitelVO Rn. 36 hält es hingegen insbesondere nicht für möglich, dass Entscheidungen, die infolge einer rügelosen Einlassung des Verbrauchers außerhalb seines Wohnsitzmitgliedstaats gem. Art. 24 EuGVVO ergangen sind entgegen Art. 6 Abs. 1 lit. d EuVTVO der EuVTVO unterfallen. Dies mag man mit der Einführung einer konstitutiven Hinweispflicht bei drohender rügeloser Einlassung von Verbrauchern gem. Art. 26 Abs. 2 EuGVVO n. F. freilich anders sehen, da hier der Verbraucher zumindest über die Folgen seiner Einlassung informiert ist.

32 Vgl. z.B. Rauscher (s.o., Fn. 19), Rn. 93; Geimer, in: Zöller (s.o., Fn. 3), Art. 6 EG-VO Europ. Vollstreckungstitel Rn. 6; Kropholler/von Hein (s.o., Fn. 3), Art. 6 EuVTVO Rn. 12; Mankowski, VuR 2010, 16, 19.

33 Vgl. etwa Stadler, in: Musielak, ZPO, 10. Aufl. 2013, Vorbemerkungen zu Art. 8-14 EuGVVO.

34 Vgl. EuGH (s.o., Fn. 1), Nr. 17-21.

35 Diese Möglichkeit besteht gem. Art. 20 Abs. 5 des Protokolls über die Satzung des Gerichtshofs vom 26. Februar 2001, ABl. C 80 v. 10.3.2001 S. 53, wenn „eine Rechtssache keine neue Rechtsfrage aufwirft.“ Hieran mochte man berechtigterweise Zweifel hegen, da zum einen die Frage der Reichweite von Art. 6 Abs. 1 lit. d EuVTVO im deutschsprachigen Schrifttum durchaus umstritten war, zum anderen klar gewesen sein musste, dass die Entscheidung weitreichende Folgen auf die Auslegung anderer Unionsrechtsakte haben würde. Ein Versäumnis, das in der teilweise überaus nachlässigen Urteilsbegründung des Gerichtshofs Niederschlag gefunden hat, s.u., D. III. und E.

36 EuGH (s.o., Fn. 1), Nr. 39.

37 EuGH (s.o., Fn. 1), Nr. 38

38 EuGH (s.o., Fn. 1), Nr. 26

39 EuGH (s.o., Fn. 1), Nr. 27

40 EuGH (s.o., Fn. 1), Nr. 29.

41 EuGH, Urt. v. 19.1.1993, Rs. C-89/91 - Shearson Lehmann Hutton Inc. ./. TVB Treuhandgesellschaft für Vermögensverwaltung und Beteiligungen $m b H$, Slg. 1993, I-139.

$42 \mathrm{EuGH}$ (s.o, Fn. 1), Nr. 32. Diese Aussage ist jedoch schlichtweg falsch, s.u., D.

43 EuGH (s.o., Fn. 1), Nr. 33

44 EuGH (s.o., Fn. 1), Nr. 37 


\section{Analyse und Kritik}

Die Folgen der Entscheidung für die EuVTVO sind klar: In C2C-Streitigkeiten ist die Bestätigung einer Entscheidung als Europäischer Vollstreckungstitel auch dann möglich, wenn dieser nicht im Wohnsitzmitgliedstaat des verklagten Verbrauchers erstritten wurde, sondern vor einem anderen mitgliedstaatlichen Gericht. Da die Grundregel gerichtlicher Zuständigkeit, wonach der Beklagte an seinem Wohnsitz verklagt werden muss, ${ }^{45}$ auch in der EuGVVO durch deren Art. 5 f. in weiten Teilen durchbrochen ist, sind Klagen an einem anderen Ort als dem Wohnsitz des Beklagten nicht nur theoretischer Natur. Die hiermit verbundenen Gefahren sind für rechtlich nicht erfahrene Privatpersonen erheblich, wie oben dargestellt. ${ }^{46}$ In der praktischen Beratung wird man seinem Mandanten, der eine unter die EuVTVO fallende Forderung in einer grenzüberschreitenden C2C-Streitigkeit tituliert erhalten hat, nur zur Bestätigung nach der EuVTVO raten können, selbst wenn nicht in mehreren Mitgliedstaaten vollstreckt werden soll, sondern nur im Wohnsitzmitgliedstaat des Schuldners. Die auf ein Minimum reduzierten Rechtsbehelfsmöglichkeiten des Schuldners und deren nahezu vollständige Verlagerung in den (entfernten) Urteilsmitgliedstaat begründen eine hohe Wahrscheinlichkeit, dass die Beitreibung der Forderung ungestört durch etwaige Einwendungen des Schuldners erfolgen kann. Dies stellt eine erhebliche Bevorzugung von Titelgläubigern dar; parallel liegende Vorschläge fanden im EuGVVO-Reformprozess politische keinen Rückhalt. ${ }^{47}$

Es ist allerdings fraglich, ob der Unionsgesetzgeber bei Streitigkeiten unter Privatpersonen diese Möglichkeit tatsächlich eröffnen wollte beziehungsweise, ob eine solche Lesart mit Blick auf die im EuGVVO-Reformprozess zutage getretene Zurückhaltung der Mitgliedstaaten gegenüber einer Einschränkung von Schuldnerrechtsbehelfen sinnhaft erscheint. Zwar hätte man entgegen dem $\mathrm{EuGH}^{48}$ vielleicht schon aus dem Wortlaut der Vorschrift des Art. 6 Abs. 1 lit. d EuVTVO folgern können, dass diese in C2C-Streitigkeiten keine Anwendung finden will: Im letzten Spiegelstrich wird gefordert, dass „der Schuldner der Verbraucher ist" ${ }^{49}$ Auch in den sonstigen Sprachfassungen findet sich die Verwendung eines bestimmten Artikels, ${ }^{50}$ derer es nicht bedurft hätte, wenn man auch C2C-Konstellationen hätte einbeziehen wollen. Denn mit einem bestimmten Artikel wird genau genommen zugleich ausgedrückt, dass die andere Partei keine Verbrauchereigenschaft besitzen kann, was bei Verwendung eines unbestimmten Artikels möglich wäre („wenn der Schuldner [ein] Verbraucher ist"). Ob dem europäischen Gesetzgeber diese sprachliche Präzision allerdings zuzutrauen ist, darf bezweifelt werden und so geht auch der EuGH hierauf nicht ein. Er stellt hingegen vor allem auf einen Vergleich der Regelung zu anderen verbraucherschützenden Vorschriften europäischen Ursprungs ab, die allesamt eine Gefällesituation im Auge hätten. Die Ausführungen des Gerichtshofs begegnen hierbei allerdings einer Reihe von Einwänden, auf die im Folgenden näher eingegangen sei.

\section{Ansetzen am Begriff "Verbraucher" verfehlt}

Obwohl das österreichische Vorlagegericht allgemein gefragt hatte, ob die Regelung des Art. 6 Abs. 1 lit. d EuVTVO eine B2C-
Konstellation fordere oder auch in einer reinen Privatstreitigkeit anwendbar sei, ${ }^{51}$ bezieht sich der Gerichtshof in seinen Ausführungen ausschließlich auf den Begriff des Verbrauchers: Es sei zu fragen, ob für die Qualifizierung einer Vertragspartei als „Verbraucher" in der fraglichen Regelung erforderlich sei, dass deren Geschäftspartner ein Unternehmer sei. ${ }^{52}$ Dies ist deshalb wenig zielführend, verliert doch ein Verbraucher nicht deshalb seine Verbrauchereigenschaft, weil er mit einem Nicht-Unternehmer Verträge schließt. Dies zeigt sich schon im 2. Spiegelstrich von Art. 6 Abs. 1 lit. d EuVTVO und auch die allgemein gebräuchliche Definition eines Verbrauchers im Unionsrecht fragt sinnvollerweise nur danach, ob eine Person einen Vertrag geschlossen hat, der nicht deren beruflicher oder gewerblicher Tätigkeit zugeordnet werden kann. ${ }^{53}$ Nähme man den EuGH beim Wort, gäbe es den Verbraucher überhaupt erst im Moment eines Vertragsschlusses mit einem Unternehmer. Mit Blick auf vorvertragliche Informationspflichten im Verbraucherprivatrecht und auch im Europäischen Zivilverfahrensrecht, erscheint diese Sichtweise allerdings unsinnig. So muss etwa das in Art. 17 EuGVVO verbriefte Verbot verbraucherbenachteiligender Prorogation sinnvollerweise schon vor Abschluss von Vertrag und Gerichtsstandsvereinbarung greifen. Hier hätte man sich, stützt sich der EuGH doch überwiegend auf eine rechtsaktübergreifende Auslegung, mehr Präzision erhoffen dürfen.

Der EuGH scheint seltsam hin- und hergerissen, was sich an seinen Ausführungen in Randnummer 24 zeigt. $^{54}$ Dennoch fokussiert er seine Ausführungen in der Folge auf den Verbraucherbegriff. Sinnvoller wäre es natürlich gewesen, die Schutzrichtung der lit. d im Gesamtkontext der Norm zu beurteilen, ein Versäumnis, das sich in sehr zweifelhaften Ausführungen des Gerichtshofs niederschlägt, was das Verhältnis der EuVTVO und des Vollstreckbarerklärungssystems der Art. 38 ff. EuGVVO angeht. ${ }^{55}$ Ebenfalls sind hierdurch weitreichende Fernwirkungen für das Zuständigkeitsrecht der EuGVVO insgesamt zu befürchten, vor allem mit Blick auf die aktuell anhängige Vorlage in der Rechtssache Kolassa. ${ }^{56}$

45 „Actor sequitur forum rei“, vgl. Art. 2 Abs. 1 EuGVVO.

46 S.o., A. I.

47 Vgl. Pohl, IPRax 2013, 109, 113.

8 EuGH (s.o., Fn. 1), Nr. 24

9 Hervorhebung durch die Verfasser.

50 Z.B. in der französischen Sprachfassung „le débiteur est le consommateur"; in der englischen "the debtor ist the consumer"; in der spanischen „el deudor sea $e l$ consumidor“; in der niederländischen „de consument de schuldenaar is"; Hervorhebung jeweils durch die Verfasser.

51 Vgl. bei EuGH (s.o., Fn. 1), Nr. 21.

52 EuGH (s.o., Fn. 1), Nr. 24.

53 Vgl. etwa Loacker, Der Verbrauchervertrag im Internationalen Privatrecht, Frankfurt a. M. 2006, S. 55 f.; Sachse, Der Verbrauchervertrag im Internationalen Privat- und Prozeßrecht, Tübingen 2006, S. 3; Mankowski, VuR 2010, 16, 20.

54 EuGH (s.o., Fn. 1), Nr. 24: „Welche Eigenschaft der Vertragspartner eines Verbrauchers haben muss [...]." Hervorhebung durch die Verfasser.

55 S.u., D. III.

56 EuGH, Rs. C-375/13 - „Kolassa“, BeckRS 2013, 80658, s.u., E. 


\section{Unanwendbarkeit von Art. 6 Abs. 1 lit. d EuVTVO mangels Gefällesituation?}

Das zentrale Argument des EuGH gegen eine Anwendung des in Art. 6 Abs. 1 lit. d EuVTVO niedergelegten Schutzes ist, dass in C2C-Streitigkeiten ein Machtgefälle nicht vorhanden, dieses aber gerade Voraussetzung für den Schutz von Verbrauchern sei. Hierauf stützen sich auch die Stimmen in der Literatur, welche nicht nur Titel aus dem Wohnsitzstaat des Verbrauchers in C2C-Konstellationen für bestätigungsfähig halten. ${ }^{57}$ Der EuGH begründet diese Sichtweise, wie bereits an anderer Stelle angedeutet, mit einer Bezugnahme auf andere europäische Rechtsakte mit verbraucherschützenden Vorschriften, welche jedoch immer nur in Gefällesituationen anzuwenden seien. ${ }^{58}$ Zwar ist es im Grundsatz richtig, dass die Privilegierung einer Partei, sei es auf materiell-rechtlicher, kollisionsrechtlicher oder verfahrensrechtlicher Ebene zugleich immer eine Abweichung von der jeweils geltenden Grundregel darstellt und daher nicht über Gebühr ausgedehnt werden darf. So schränkt die Sonderanknüpfung von Verbraucherverträgen in Art. 6 Rom I-VO die grundsätzlich gem. Art. 4 Rom I-VO bestehende Rechtswahlfreiheit der Parteien ein. Gleiches gilt für die $\mathrm{Zu}$ ständigkeitsvorschrift des Art. 16 Abs. 1 EuGVVO, mit der die in Art. 2 Abs. 1 EuGVVO verbriefte Grundregel des actor sequitur forum rei bei Aktivklagen eines Verbrauchers gegenüber einem Unternehmer außer Kraft gesetzt wird.

\section{Schutz durch Art. 6 Abs. 1 lit. d EuVTVO tatsächlich Frage einer Gefällesituation?}

Dennoch erscheint die Beschränkung der Schutzvorschrift des Art. 6 Abs. 1 lit. d EuVTVO auf Gefällesituationen nicht zwingend. ${ }^{59}$ Diese auf Ebene der (Vorbereitung der) Zwangsvollstreckung angesiedelte Weichenstellung muss nicht zwingend parallel ausgelegt werden zu zuständigkeitsrechtlichen Verbraucherschutzvorschriften, auf die sich der EuGH unter anderem bezieht. Vor allem erscheint sein Verweis auf den in Art. 16 EuGVVO angesiedelten Verbrauchergerichtsstand, der natürlich nur in Fällen eines Machtgefälles einschlägig ist, verfehlt. ${ }^{60} \mathrm{Zum}$ einen hätte der EuGH sich davor in systematischer Auslegung zunächst mit Art. 6 Abs. 1 lit. b EuVTVO auseinandersetzen müssen, der im Unterschied zu lit. d (ausdrücklich) an die in der EuGVVO vertypten Gefällesituationen im Verhältnis Arbeitgeber/Arbeitnehmer und Versicherung/Versicherter anknüpft. Zum anderen fragt sich in den hier interessierenden Fällen, im Gegensatz etwa zu einer zuständigkeitsrechtlichen Privilegierung über Art. 16 Abs. 1, 2 EuGVVO - die in C2C-Streitigkeiten logischerweise nicht durchführbar ist ${ }^{61}$ - auf einer sekundären Ebene nur, ob genau hier der Schutz von Privatpersonen vor den Folgen eines Europäischen Vollstreckungstitels sinnvollerweise gewährt werden sollte.

Dies ist eine Frage, die unabhängig von der Person des Vertragspartners hätte beantwortet werden können. Sieht man Art. 6 Abs. 1 lit. d EuVTVO nicht nur als Vorschrift, die eine fehlerhafte Zuständigkeitsannahme entgegen Art. 16 EuGVVO durch das Ursprungsgericht im Bestätigungsverfahren wieder ausbügeln soll, ${ }^{62}$ hätte man hierauf aufbauend eine an den Interessen der Beteiligten ausgerichtete Beschränkung der Verordnung erreichen können. Denn Folge der Bestätigung als Europäischer Vollstreckungstitel ist nicht nur eine Verschiebung der Rechtsbe- helfsmöglichkeiten in den Ursprungsmitgliedsstaat des Urteils als gewissermaßen zuständigkeitsrechtliche Frage ${ }^{63}$-, sondern vor allem auch eine Beschränkung der Anfechtungsmöglichkeiten des Titelschuldners in der Sache. Warum dies für eine Privatperson in den Fällen der Inanspruchnahme durch eine andere Privatperson tragbar sein soll, in Fällen der Inanspruchnahme durch einen Unternehmer dagegen untragbar, ist schwer zu begründen.

Wird beispielsweise ein Verbraucher von einem Taxiunternehmer außerhalb seines Wohnsitzmitgliedstaats wegen Ansprüchen aus einem grenzüberschreitenden Beförderungsvertrag $^{64}$ verklagt und erfolgt im Verfahren eine Verletzung des verfahrensrechtlichen ordre publics - es wird dem Verbraucher z.B. willkürlich keinerlei rechtliches Gehör im Verfahren gewährt, obwohl er sich tatsächlich vor Gericht verteidigen möchte ${ }^{65}-$, könnte der Verbraucher bei einem Vorgehen nach der EuGVVO Art. 34 Nr. 1 EuGVVO bzw. Art. 45 Abs. 1 lit. a EuGVVO n. F. einwenden. Wollte der Unternehmer hier nach der EuVTVO vorgehen, wäre dies wegen Art. 6 Abs. 1 lit. d EuVTVO nicht möglich. Der Schuldner wäre also in jedem Fall vor der Vollstreckung eines eklatanten Fehlurteils geschützt. Ersetzt man den klagenden Unternehmer durch eine Privatperson, wäre eine Bestätigung der Entscheidung nach der EuVTVO möglich. Damit entfiele für den Schuldner jegliche Möglichkeit der Verteidigung in der Zwangsvollstreckung und dies, obwohl er sich in das ausländische Verfahren tatsächlich einbringen wollte. Die Person des Klägers ist für ihn beide Male völlig irrelevant, das Ergebnis jeweils diametral entgegengesetzt. Damit erscheint es zweifelhaft, ob Art. 6 Abs. 1 lit. d EuVTVO tatsächlich nur ein Machtgefälle zwischen den Parteien zum Bezugspunkt hat bzw. nur als Korrekturvorschrift für eine Verkennung von Art. 16 EuGVVO durch das Ursprungsgericht darstellt, was insbesondere dann zweifelhaft erscheint, wenn die Vorschrift, wie im Beispiel, etwa wegen Art. 15 Abs. 3 EuGVVO offensichtlich gar nicht zur Anwendung gelangen will.

Diesbezüglich sei noch die Frage erlaubt, wie der EuGH entscheiden würde, wenn eine in einem C2C-Rechtsstreit außerhalb

57 Etwa Bach (s.o., Fn. 3), S. 220; Kropholler/von Hein (s.o., Fn. 3), Art. 6 EuVTVO Rn. 13.

58 S.o., C.

59 So Mankowski, VuR 2010, 16, 19 f., i.E. aber ablehnend.

60 Vgl. EuGH (s.o., Fn. 1), Nr. 34.

61 Vgl. Mankowski, VuR 2010, 16, 20: Der zwingende Gerichtsstand bei Klagen gegen einen Verbraucher an dessen Wohnsitz gem. Art. 16 Abs. 2 EuGVVO würde mit dem Klägergerichtsstand des klagenden Verbrauchers gem. Art. 16 Abs. 1 EuGVVO in einen unauflösbaren Konflikt geraten.

62 So Bach (s.o., Fn. 3), S. 219.

63 Diesbezüglich könnte man eine Parallele zu den Art. 15 ff. EuGVVO ziehen und vorbringen, dass eine Rechtsverfolgung im Ausland eben nur dann für einen Verbraucher billigerweise unzumutbar sein soll, wenn ihm ein Unternehmer als Partei gegenübersteht, da von dieser typischerweise eine gewisse Abschreckungswirkung auf Verbraucher ausgeht bzw. ihm eine Prozessführung im Ausland eher zugemutet werden kann.

64 Für derartige Verträge ist der Verbrauchergerichtsstand gem. Art. 15 Abs. 3 EuGVVO nicht eröffnet.

65 Dass derartige Fälle auch im Europäischen Justizraum leider Realität sind, zeigt z.B. BGHZ 182, 188: Vaterschaftsfeststellung durch ein polnisches Gericht nur auf Grundlage der Aussage einer Zeugin vom Hörensagen, keine Einholung eines Sachverständigengutachtens und Ablehnung des Angebots des mutmaßlichen Vaters, freiwillig an einem Gentest zur Bestimmung der Abstammung mitzuwirken. 
des Wohnsitzmitgliedstaats des verklagten Verbrauchers titulierte Forderung an einen Unternehmer übertragen würde und dieser im Ursprungsmitgliedstaat des Titels die Bestätigung nach der EuVTVO zu beantragen suchen würde. Wäre der Verbraucher hier zu schützen? Ein Machtgefälle im Zeitpunkt der Bestätigung bestünde jedenfalls. Oder kommt es alleine auf den Zeitpunkt der Titulierung an? Und wie wäre es im umgekehrten Fall: Wäre Art. 6 Abs. 1 lit. d EuVTVO auch dann anzuwenden, wenn ein Verbraucher in Verkennung der Zuständigkeitsvorschriften der Art. 15 ff. EuGVVO von einem Unternehmer außerhalb seines Wohnsitzmitgliedstaats verklagt würde und Letzterer nach Titulierung die Forderung an einen Verbraucher übertrüge, der eine Bestätigung nach der EuVTVO beantragen würde? Die Beispiele zeigen, dass durch eine von Gefällesituationen losgelöste Betrachtung der Vorschrift gewisse Folgeprobleme hätten vermieden werden können.

\section{Unbillige Benachteiligung des klagenden Verbrauchers?} Hätte der EuGH Art. 6 Abs. 1 lit. d EuVTVO auf C2C-Streitigkeiten erstreckt, wären klagende Verbraucher jedenfalls nicht über Gebühr belastet. ${ }^{66}$ Bach etwa meint zwar, der klagende Verbraucher sei besonders hart davon getroffen, müsse er doch den Weg einer Vollstreckbarerklärung im Ausland gehen. ${ }^{67}$ Spätestens mit dem Inkrafttreten der reformierten EuGVVO wird dieses Argument jedoch nicht mehr überzeugen. Würde sich ein Gläubiger dazu entschließen, ein Erkenntnisverfahren außerhalb des Wohnsitzmitgliedstaats eines schuldenden Verbrauchers durchzuführen, müsste er zwar derzeit noch das Vollstreckbarerklärungsverfahren der EuGVVO durchlaufen. Ab Anfang 2015 wird allerdings nicht einmal mehr das notwendig sein. Ein - billigerweise zu berücksichtigender ${ }^{68}$ - Nachteil zur Bestätigung nach der EuVTVO wird hier nicht mehr gegeben sein. Wäre dem Gläubiger daran gelegen, einen Europäischen Vollstreckungstitel gegen den anderen Verbraucher zu erlangen, müsste er diesen an seinem allgemeinen Gerichtsstand verklagen, was das sollte nicht vergessen werden! - der allgemein konsentierten Grundregel des actor sequitur forum rei entspricht. Durch die regelmäßige Beschränkung von Privatvermögen auf den jeweiligen Wohnsitzmitgliedstaat, besteht in C2C-Streitigkeiten auch kein wirkliches Bedürfnis für den Gläubiger, ein im Ausland erstrittenes Urteil als Europäischen Vollstreckungstitel bestätigen zu lassen. ${ }^{69}$ Wollte er sich dennoch die Vorteile der EuVTVO sichern, um etwa in das im Ausland belegen Ferienhaus seines Schuldners erleichtert vollstrecken zu können, ${ }^{70}$ müsste er an dessen Wohnsitz klagen, ${ }^{71}$ was insoweit interessengerecht erscheint. Zugegebenermaßen wäre mit einer derartigen Sichtweise der Anwendungsbereich der EuVTVO teils erheblich eingeschränkt worden. Gerade der mangelnde politische Konsens im EuGVVO-Reformprozess zeigt jedoch, dass eine sinnvolle Beschränkung der EuVTVO durch den EuGH wohl nicht auf erhebliche Kritik gestoßen wäre.

\section{Notwendigkeit dieser Auslegung zur Verhinderung von Friktionen zwischen EuGVVO und EuVTVO?}

Schließlich hält der EuGH die von ihm vorgeschlagene Auslegung auch deshalb für notwendig, um Verwerfungen zum Vollstreckbarerklärungssystem der Art. 38 ff. EuGVVO zu verhindern, welches durch die EuVTVO ergänzt werde. ${ }^{72}$ Hier wird dem EuGH das oben ${ }^{73}$ kritisierte Anknüpfen an den Begriff des Verbrauchers zum Verhängnis: Es wäre widersprüchlich, wenn in C2C-Streitigkeiten die Bestätigung anderer Titel als solcher aus dem Wohnsitzmitgliedstaat des verklagten Verbrauchers nach der EUVTVO auszuscheiden habe, aber trotzdem eine Vollstreckbarerklärung nach der EuGVVO erlangt werden könne, gegen die eine Verletzung der Art. 15 ff. EuGVVO nicht vorgebracht werden könne. ${ }^{74}$ Dieses Argument ist geradezu absurd: Es ist in keiner Weise widersprüchlich, wenn ein Titelgläubiger das Exequatur nach der EuGVVO erlangen kann, aber ein Vorgehen nach der EuVTVO ausgeschlossen ist. Das Vollstreckbarerklärungsverfahren nach der EuGVVO steht im Rahmen seines Anwendungsbereiches immer zur Verfügung. Die EuVTVO als speziellere Verordnung findet dagegen nur unter besonderen Voraussetzungen Anwendung, da sie auch eine erhebliche Einschränkung der Beklagtenrechte mit sich bringt. Dass diese nicht immer zur Anwendung kommen kann, ist ihr damit wesenseigen. Würde man daher Art. 6 Abs. 1 lit. d EuVTVO auch auf C2C-Streitigkeiten erstrecken, wäre es ohne jeglichen Widerspruch in der Sache, dem Titelgläubiger die Vollstreckbarerklärung nach der EuGVVO zu ermöglichen - der Einwand der Verletzung von Art. 16 Abs. 2 EuGVVO wäre gerade nicht möglich, da dieser auf B2C-Streitigkeiten zugeschnitten ist - eine Bestätigung nach der EuVTVO aber zu verwehren.

\section{E. Ausblick}

Die Entscheidung des EuGH könnte sich auf lange Sicht auf die derzeit anhängige Vorlage in der Rechtssache Kolassa ${ }^{75}$ auswirken. Hier wurde der Gerichtshof unter anderem danach gefragt, ob sich ein Verbraucher auch dann auf die Regeln der Art. 15 ff. EuGVVO berufen kann, wenn er eine Inhaberschuldverschrei-

66 A.A. Bach (s.o., Fn. 3), S. 221; Kropholler/von Hein (s.o., Fn. 3), Art. 6 EuVTVO Rn. 13 m.w.N.

67 Bach (s.o., Fn. 3), S. 221

68 Natürlich mag man einwenden, bei einer Bestätigung nach der EuVTVO sei es dem Titelschuldner in der Folge nicht mehr möglich, etwa eine Verletzung des ordre public im Staate der Zwangsvollstreckung zu rügen, was für den Gläubiger letztlich von Vorteil sei. Ob allerdings die Verletzung fundamentaler Rechtsgrundsätze - etwa Verfahrensgarantien - als Ausgangspunkt für ein Argument dienen sollten, ist in hohem Maße fragwürdig.

69 Vgl. Heringer (s.o., Fn. 3), S. 118; Mankowski, VuR 2010, 16, 19.

70 Beispiel bei Stein, IPRax 2004, 181, 189.

71 Vgl. dazu Stadler, RIW 2004, 801, 804.

72 EuGH (s.o., Fn. 1), Nr. 35-37. Auch Mankowski, VuR 2010, 16, 21 verweist darauf, dass Friktionen zu benachbarten Rechtsakten zu vermeiden sein. Welche Rechtsakte genau gemeint sind und worin etwaige Friktionen bestehen sollen, wird aber nicht gesagt.

73 S.o., D. I.

74 EuGH (s.o., Fn. 1), Nr. 37.

75 EuGH, Rs. C-375/13 - „Kolassa“, BeckRS 2013, 80658. 
bung erworben hat und so in einen zwischen zwei Unternehmern abgeschlossenen Vertrag eintritt. In der der vorliegend besprochen Entscheidung verweist der EuGH auf das Urteil in Sachen Shearson Lehman Hutton ${ }^{76}$ und behauptet, hier habe er entschieden, dass ein zwischen zwei Unternehmern abgeschlossener Vertrag nicht in den Anwendungsbereich der verbraucherschützenden Vorschriften des EUGVÜ bzw. jetzt der EuGVVO falle. ${ }^{77}$ Dies ist jedoch schlicht falsch. Der Entscheidung Shearson Lehman Hutton lag eine Abtretungskonstellation zugrunde: Ein Verbraucher hatte seinen aus einem B2C-Vertrag herrührenden Anspruch an eine gewerblich handelnde Gesellschaft angetreten, die im Ergebnis richtigerweise nicht in den Genuss eines Klägergerichtsstandes kam. ${ }^{78}$ Die Bezugnahme in Vapenik stellt demnach entweder eine unbewusste Fehlleistung oder eine bewusste Verdrehung von Tatsachen dar, wobei beide Möglichkeiten bedenklich erscheinen. Es bleibt daher zu hoffen, dass der EuGH in seiner Entscheidung in Kolassa unabhängig von seinen Ausführungen in Vapenik zu einem sinnvollen Urteil gelangt und sich nicht auf eine „mittlerweile gefestigte Rechtsprechung“ zurückzieht, wonach zwischen Unternehmern abgeschlossene Verträge niemals in den Anwendungsbereich der Art. 15 ff. EuGVVO fielen. ${ }^{79}$

\section{F. Zusammenfassung}

Die EuVTVO ist nach der Entscheidung des EuGH in der Rechtssache Vapenik in C2C-Streitigkeiten auch auf solche Titel anwendbar, die außerhalb des Wohnsitzmitgliedstaates des verklagten Verbrauchers erstritten wurden. Damit wird die Prozessführungslast von Privatpersonen im Ausland erheblich erhöht. Vor dem Hintergrund der gescheiterten Straffung der EuGVVO in diesem Bereich, hätte man durchaus auch eine hieran orientierte Auslegung der EuVTVO wählen können. Daneben könnte sich die Entscheidung ungünstig auf die Beurteilung der Vorlage in Sachen Kolassa auswirken, was abzuwarten bleibt.

\section{Summary}

According to the ECJ the European enforcement order regulation also applies to judgments in c2c-disputes which were delivered by courts outside the place where the consumer sued is domiciled. Private persons are thus faced with an increased obligation to defend themselves outside their domicile. In regard to the recent reforms concerning Brussels I, this development appears questionable. Besides, the judgment may affect the preliminary reference procedure in the case Kolassa.

\section{Résumé}

Suivant l'arrêt de la CJE dans l'affaire Vapenik, le règelement d'un titre exécutoire européen applique en cas d'une dispute entre consommateurs même si le jugement était rendu en dehors de l'État membre de résidence du débiteur. En conséquence, la nécessité de se défendre à l'étranger est élevé. En regard de la révision du Bruxelles I, ce développement est discutable. En plus, l'arrêt pourrais affecter la procédure préjudicielle dans l'affaire Kolassa.

76 EuGH, Urt. v. 19.1.1993, Rs. C-89/91 - Shearson Lehmann Hutton Inc. ./. TVB Treuhandgesellschaft für Vermögensverwaltung und Beteiligungen $m b H$, Slg. 1993, I-139.

77 EuGH (s.o., Fn. 1), Nr. 32.

78 Vgl. EuGH (s.o., Fn. 76), 183, Nr. 3.

79 In Kolassa könnte man etwa den Vertragseintritt des Verbrauchers selbst als Verbrauchervertrag einordnen, wenn und da dieser mit Billigung des anderen Vertragsteils erfolgen muss. 